\title{
Coronaviral hypothetical and structural proteins were found in the intestinal surface enterocytes and pneumocytes of severe acute respiratory syndrome (SARS)
}

Wai S Chan ${ }^{1}$, Chun $\mathrm{Wu}^{2}$, Sammy CS Chow ${ }^{3}$, To Cheung ${ }^{3}$, Ka-Fai To ${ }^{1}$, Wai-Keung Leung ${ }^{4}$, Paul KS Chan ${ }^{5}$, Kam-Cheong Lee ${ }^{6}$, Ho-Keung Ng${ }^{1}$, Deborah MY Au ${ }^{3}$ and Anthony WI Lo ${ }^{1}$

${ }^{1}$ Department of Anatomical and Cellular Pathology, The Chinese University of Hong Kong, Hong Kong SAR, China; ${ }^{2}$ Abgent Inc., San Diego, CA, USA; ${ }^{3}$ Century Biotech Limited, Hong Kong SAR, China; ${ }^{4}$ Department of Medicine and Therapeutics, The Chinese University of Hong Kong, Hong Kong SAR, China; ${ }^{5}$ Department of Microbiology and School of Public Health, The Chinese University of Hong Kong, Hong Kong SAR, China and ${ }^{6}$ Department of Pathology, Princess Margaret Hospital, Hong Kong SAR, China

\begin{abstract}
Severe acute respiratory syndrome (SARS) is a newly emerging infectious disease that haunted the world from November 2002 to July 2003. Little is known about the biology and pathophysiology of the novel coronavirus that causes SARS. The tissue and cellular distributions of coronaviral hypothetical and structural proteins in SARS were investigated. Antibodies against the hypothetical (SARS 3a, 3b, 6, 7a and 9b) and structural proteins (envelope, membrane, nucleocapsid and spike) of the coronavirus were generated from predicted antigenic epitopes of each protein. The presence of these proteins were first verified in coronavirus-infected Vero E6 tissue culture model. Immunohistochemical studies on different human tissues, including a cohort of nine autopsies, two liver biopsies and intestinal biopsies of SARS patients, further confirmed the existence of coronaviral hypothetical and structural proteins in the cytoplasm of pneumocytes and small intestinal surface enterocytes in SARS patients. With this vast array of antibodies, no signal was observed in other cell types including those organs in which reverse transcriptase-polymerase chain reactions were reported to be positive. Structural proteins and the functionally undefined hypothetical proteins were expressed in coronavirusinfected cells with distinct expression pattern in different organs in SARS patients. These antipeptide antibodies can be useful for the diagnosis of SARS at the tissue level.
\end{abstract}

Modern Pathology (2005) 18, 1432-1439. doi:10.1038/modpathol.3800439; published online 13 May 2005

Keywords: SARS; coronavirus; immunohistochemistry

From November 2002 to July 2003, severe acute respiratory syndrome (SARS) had affected more than 8000 people in 28 regions worldwide with mortality upto $9.6 \% .^{1}$ The clinical picture of this imminent infectious disease was dominated by respiratory system involvement. ${ }^{2,3}$ Gastrointestinal symptoms were also common. ${ }^{4,5}$

The primary etiological agent of SARS is a novel coronavirus (SARS-CoV) ${ }^{6-8}$ The genome of SARS$\mathrm{CoV}$ consists of a single giant positive-strand RNA

Correspondence: Dr AWI Lo, MBChB, PhD, Department of Anatomical and Cellular Pathology, The Chinese University of Hong Kong, Prince of Wales Hospital, 30-32 Ngan Shing Street, Shatin, New Territories, Hong Kong SAR, China.

E-mail: awilo@cuhk.edu.hk

Received 9 January 2005; revised 16 March 2005; accepted 17 March 2005; published online 13 May 2005 that is approximately $29.7 \mathrm{~kb}$ in length. SARS-CoV genome encompasses two large open reading frames (ORFs) encoding nonstructural proteins involved in replication and 12 potential ORFs. These potential ORFs include four genes encoding structural proteins (envelope, membrane, nucleocapsid and spike proteins). The remaining potential ORFs encode hypothetical SARS-CoV-specific proteins that lack obvious sequence similarity to known proteins..$^{9,10}$ These hypothetical proteins that are purely the prophecies of bioinformatics algorithm need to be studied in infected cells so as to confirm their identities. The functions of these hypothetical proteins and their roles in SARS pathogenesis remain obscure. ${ }^{11,12}$

In this study, we have generated specific antipeptide antibodies against each of the putative structural and hypothetical proteins of the SARS-CoV. 
Using immunohistochemistry, we demonstrated the unique existence of corresponding peptide sequences in SARS-CoV-infected tissue culture models and clinical specimens. The tissue and cellular distribution pattern of SARS-CoV hypothetical and structural proteins in SARS was studied.

\section{Materials and methods}

\section{Selection of Clinical Specimens}

Clinical specimens were selected retrospectively following the guidelines of the local ethics committee. SARS was diagnosed according to the criteria of World Health Organization. ${ }^{1}$ The outbreak in the Prince of Wales Hospital, Hong Kong, and the sequence of events leading to the world endemic has been reported. ${ }^{2,3}$ Pathology of Case 1 to 8 has been previously described. ${ }^{13}$ One probable SARS case (negative culture) during the same epoch and two lung sections from autopsies performed in early 2002 (with the diagnosis unrelated to lung diseases) were used as negative controls. The terminal ileum and colon biopsies ${ }^{5}$ of one SARS patient and liver biopsies ${ }^{14}$ were obtained with informed consents. In all, 10 cases of randomly selected autopsies, with causes of death either related or unrelated to pulmonary pathology, in the pre-SARS era of 2000-2001 were used as negative control.

\section{Generation of Antipeptide Antibodies}

Antigenicities of epitopes on putative ORF of the SARS-CoV (Genbank accession no.: NC_004718) were evaluated in silico using Clone Manager 5 (Scientific \& Educational Software), Peptide Companion (CSPS Pharmaceuticals, Inc.) and DS Gene (Accelrys). These peptides were also compared with other published proteomes of coronavirus (CoV) such as human Group 1 and Group $2 \mathrm{CoV}$, Bovine$\mathrm{CoV}$, Porcine-CoV, Feline-CoV and Canine-CoV to select peptides that were SARS-CoV-specific. Antibodies were generated from rabbits immunized with each individual keyhole limpet hemocyanin-conjugated synthetic peptide. All antipeptide antibodies and peptides used in this study were produced by Abgent (San Diego, CA, USA) and are now commercially available at Abgent.

\section{Cell Lines and SARS-CoV Infection}

The cell line Vero E6 (monkey kidney cell line) was obtained from the American Type Culture Collection (Manassas, VA, USA). The virus CUHK-W1 strain of SARS-CoV (GenBank accession no.: AY278554) was grown and assayed in the cell line as previously described. ${ }^{15}$

\section{Immunohistochemical Studies}

Paraffin blocks were prepared from infected or control Vero E6 fixed with 10\% formalin as routine clinical cytology specimens. Autopsy and biopsy specimens were fixed with $10 \%$ formalin and embedded in paraffin blocks. Standard avidinbiotin method was used for immunohistochemical studies on $4-\mu \mathrm{m}$ sections from selected paraffin blocks. Antipeptide antibodies (1:100) with StreptABComplex/HRP Duet Reagent Set (DAKO, Glostrup, Denmark) were used according to the manufacturer's protocols with 3, 3'-diaminobenzidine tetrahydrochloride as the chromogen. Antigen retrieval was performed by microwave pretreatment twice in $10 \mathrm{mM}$ citrate buffer, pH 6.0 or $0.1 \mathrm{M}$ EDTA buffer, $\mathrm{pH}$ 8.0, with preliminary heating at $780 \mathrm{~W}$ for $3 \mathrm{~min}$ followed by $480 \mathrm{~W}$ for $10 \mathrm{~min}$. The specificity of each antibody for immunohistochemistry was ascertained in formalin-fixed Vero E6 cells by suppression of signals when the primary antibody was preincubated with excess corresponding peptides before applying to the cell block sections. Intensities of the immunohistochemical signals on each individual cell were judged arbitrarily with the overall assessment as: negative $(-)$, positive $(+)$, moderately positive $(++)$ or markedly positive $(+++)$.

\section{Immunofluorescence-Fluorescence In Situ Hybridization Studies}

The coexistence of SARS-CoV protein and RNA in infected cells was detected by immunofluorescence-fluorescence in situ hybridization on formalin-fixed paraffin-embedded tissues as previously described. ${ }^{16}$ A 700 -bp reverse transcriptase-polymerase chain reaction product corresponding to the M gene of SARS-CoV strain Su10 (Genbank accession no.: AY282752) was used as the probe. Antipeptide antibodies (1:100) were used for colocalization. Fluorescence in situ hybridization signals were detected using anti-DIG-rhodamine (Roche) and the secondary antibody for immunofluorescence was antibiotin fluorescein thiocyanate (FITC). Nuclei were counterstained with 4,6-diamidino-2-phenylindole in antifade mountant (Vectorshield, Vector Laboratories).

\section{Results}

\section{Coronaviral Hypothetical and Structural Proteins were Detected in the Cytoplasm of Culture Cells Infected by SARS-CoV}

Antigenicity of epitopes of the putative hypothetical and structural proteins was evaluated and anti-peptide antibodies were generated in rabbits (Table 1). Putative epitopes were identified in nine out of 14 predicted ORFs, representing $64 \%$ of the transcriptome, of SARS-CoV. These antibodies for immunohistochemical analysis were evaluated in vitro on Vero E6 infected by SARS-CoV. Uninfected cells were used as negative controls. All epitopes of hypothetical (SARS 3a, 3b, 6, 7a and 9b) and 
Table 1 Immunohistochemical studies of antipeptide antibodies on Vero E6 cells infected by SARS-CoV

\begin{tabular}{|c|c|c|c|c|}
\hline Protein $^{\mathrm{a}}$ & $\begin{array}{l}\text { Peptide } \\
\text { position }^{\mathrm{b}}\end{array}$ & Antibody & Vero & Background \\
\hline Envelope & $60-76$ & SARS-AbS18a & $++^{\mathrm{c}}$ & + \\
\hline \multirow[t]{2}{*}{ Membrane } & 1-18 & SARS-AbS17a & + & - \\
\hline & $204-221$ & SARS-AbS16b & +++ & - \\
\hline \multirow[t]{10}{*}{ Spike } & 1135-1150 & SARS-AbS1a & + & - \\
\hline & $1158-1171$ & SARS-AbS2 & - & - \\
\hline & 899-912 & SARS-AbS3b & - & - \\
\hline & $785-799$ & SARS-AbS4b & - & - \\
\hline & $540-554$ & SARS-AbS5a & +++ & - \\
\hline & $439-454$ & SARS-AbS6b & + & - \\
\hline & $204-219$ & SARS-AbS7b & ++ & - \\
\hline & $24-43$ & SARS-AbS8a & + & + \\
\hline & $1236-1255$ & SARS-AbS23b & + & - \\
\hline & 19-38 & SARS-AbS24a & ++ & - \\
\hline \multirow[t]{2}{*}{ Nucleocapsid } & $406-422$ & SARS-AbS12a & ++ & - \\
\hline & $1-17$ & SARS-AbS13a & +++ & - \\
\hline \multirow[t]{2}{*}{ SARS 3a } & $258-275$ & SARS-AbS21a & +++ & - \\
\hline & $134-153$ & SARS-AbS22b & - & - \\
\hline \multirow[t]{2}{*}{ SARS 3b } & $31-45$ & SARS-AbS20b & + & - \\
\hline & $135-154$ & SARS-AbS19a & ++ & - \\
\hline SARS 6 & $45-63$ & SARS-AbS15b & + & + \\
\hline \multirow{2}{*}{ SARS 7a } & 85-104 & SARS-AbS14b & - & - \\
\hline & $35-54$ & SARS-AbS9b & + & - \\
\hline \multirow[t]{2}{*}{ SARS 9b } & $28-44$ & SARS-AbS11 & ++ & - \\
\hline & $78-97$ & SARS-AbS10b & ++ & - \\
\hline
\end{tabular}

${ }^{\mathrm{a}}$ Proteins are named according to the reference genome sequence of SARS-CoV (Genbank: NC_004718).

${ }^{\mathrm{b}}$ Positions of peptides are labeled relative to individual ORFs of SARS-CoV.

C $_{+}$' indicates positive by immunohistochemistry (graded into + , moderate '++', and marked ' +++ '); '-' indicates negative by immunohistochemistry. structural proteins (envelope, membrane, nucleocapsid and spike) were detected in the cytoplasm of infected Vero E6 cells by at least one antibody from each protein (Table 1). Intensities of the signals in individual infected cells were scored arbitrarily. Nuclear signals were not detected. A similar cytoplasmic signal was not observed in uninfected Vero E6 negative controls. Representative results with the antibody SARS-AbS13a are shown in Figure 1. Hence, the peptide sequences of each putative hypothetical and structural protein were expressed in vitro. Some of the epitopes, although theoretically antigenic, might be buried in the interior of the proteins. The antipeptide antibodies with strong cytoplasmic signals and minimal background were further applied on formalin-fixed paraffin-embedded sections of clinical specimens.

\section{SARS-CoV Hypothetical and Structural Proteins are Detected in the Cytoplasm of Intestinal Surface Enterocytes and Pneumocytes}

Antibodies SARS-AbS13a, SARS-Abs-16b, SARSAbs-5a and SARS-AbS21a, against the nucleocapsid protein $(\mathrm{N})$, the membrane protein $(\mathrm{M})$, the spike protein (S) and a putative SARS 3a protein, respectively, were selected for further evaluation in 16 different tissues, including the lung, bronchus, small intestine, stomach, colon, pancreas, lymph node, spleen, bone marrow, liver, kidney, adrenal gland, skeletal muscle, heart and skin, from nine fatal cases of SARS (Table 2). Terminal ileum and colon biopsies from one patient ${ }^{5}$ and two liver

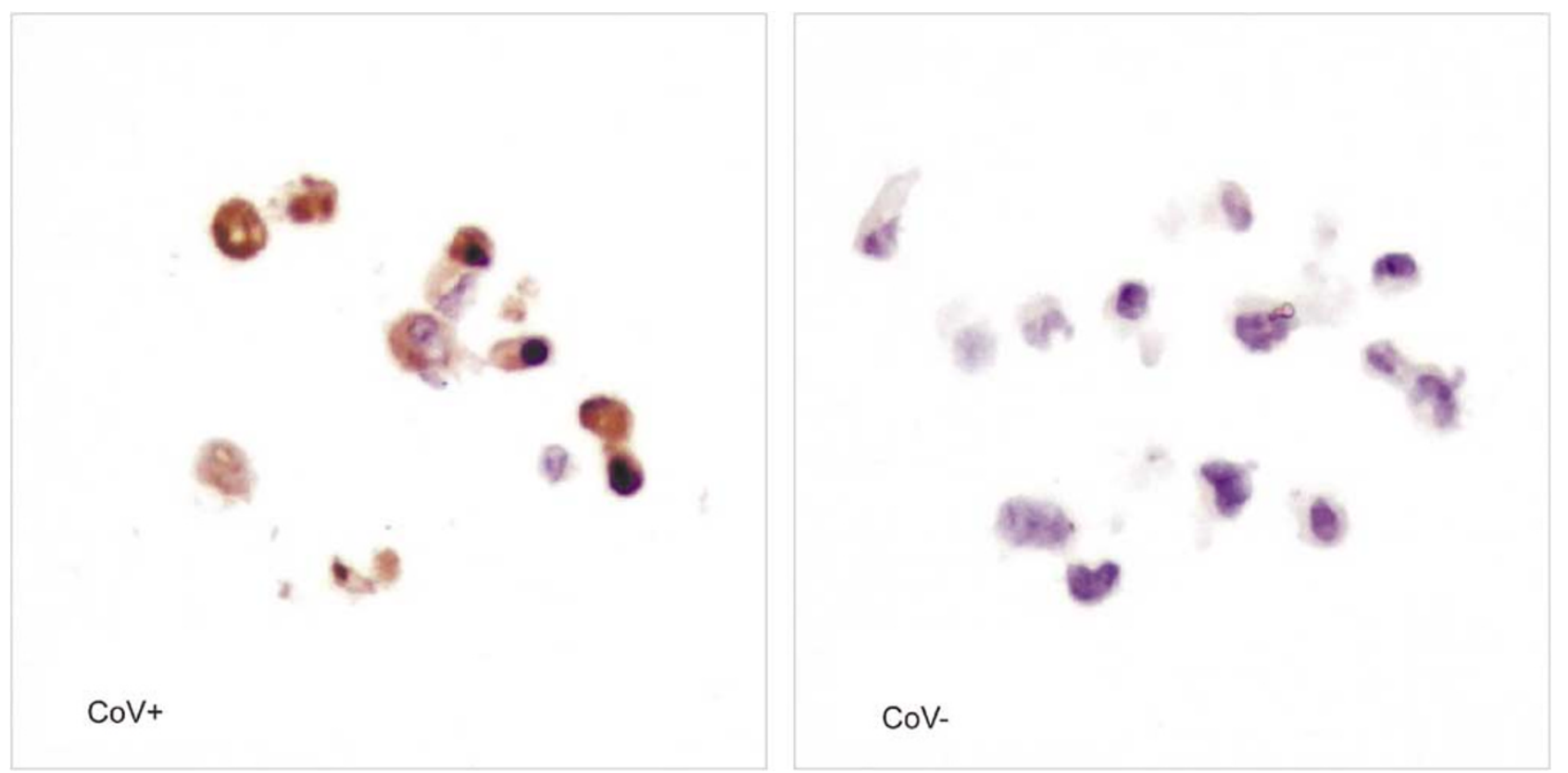

Figure 1 Immunohistochemical studies of antipeptide antibody SARS-AbS13a against the nucleocapsid protein on SARS-CoV-infected $(\mathrm{CoV}+)$ and uninfected (CoV-) culture of Vero E6. Positive cytoplasmic signals were shown in brown color. The nuclei were counterstained blue by hematoxylin $(\times 400)$. 
Table 2 Results of immunohistochemical analysis on SARS autopsy cases

\begin{tabular}{|c|c|c|c|c|c|c|c|c|c|}
\hline & Case 1 & Case 2 & Case 3 & Case 4 & Case 5 & Case 6 & Case 7 & Case 8 & Case 5 \\
\hline Lung culture & + & + & - & - & - & - & - & + & - \\
\hline Lung IHC: N & + & + & - & - & - & - & - & + & - \\
\hline Lung IHC: SARS 3a & + & + & - & - & - & - & - & + & - \\
\hline Lung ISH & + & + & - & - & - & - & - & + & - \\
\hline Lung EM & + & + & + & + & - & + & + & + & - \\
\hline SI culture & + & + & + & - & + & + & NA & NA & - \\
\hline SI IHC: N & + & + & + & - & + & + & NA & NA & - \\
\hline SI IHC: SARS 3a & + & + & + & - & + & + & NA & NA & - \\
\hline SI ISH & + & + & + & - & + & + & NA & NA & - \\
\hline SI EM & + & + & + & + & - & + & NA & NA & - \\
\hline
\end{tabular}

EM: electron microscopy; ISH: in situ hybridization; IHC: immunohistochemistry; SI: small intestine; +: positive result; -: negative results; N: nucleocapsid protein; NA: not available.

Bronchus, stomach, colon, pancreas, lymph node, spleen, bone marrow, liver, kidney, adrenal gland, skeletal muscle, heart and skin samples from the above nine cases were immunohistochemical-negative.
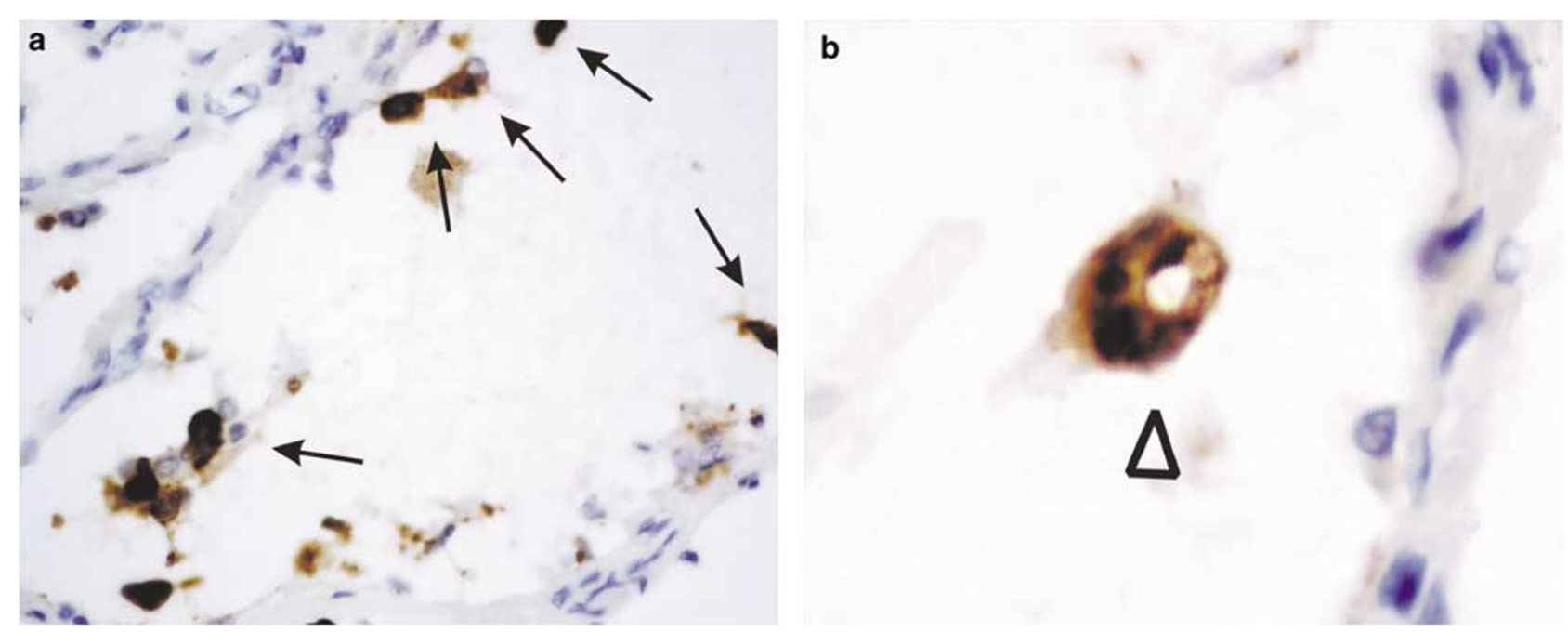

Figure 2 Immunohistochemical studies of antipeptide antibody SARS-AbS13a against the nucleocapsid protein on lung autopsy sections. SARS-CoV-infected cells were demonstrated by the strong cytoplasmic signals in pneumocytes (a and b, arrows) and multinucleated giant cells (b, arrow head). The sections were counter-stained with hematoxylin $(\times 400)$.

biopsies ${ }^{14}$ from different patients were also included in this study. Positive immunohistochemical signals were observed in the lung sections from three fatal cases and the small intestine sections from five autopsy cases. SARS-CoV proteins were detected only in samples that were positive for viral culture and viral genome. Other tissue samples were all immunohistochemically negative. The SARS-CoV proteins existed either in diffuse signals or in aggregates in the cytoplasm. Background in negative controls was minimal. Immunohistochemical staining was negative in the lung and intestinal sections from control autopsy and surgical specimens.

The histopathology of the lung was dominated by diffuse alveolar damage at various stages of organization. No histological features appeared to predict the positivity of immunohistochemistry. A similar result was obtained previously for in situ hybridization study of the SARS-CoV genome. ${ }^{16}$ In immuno- histochemical-positive lung sections, positive signals were observed in the cytoplasm of pneumocytes lining the alveolar septa (Figure 2a, arrows) and in the detached pneumocytes within the alveolar spaces (Figure 2a, arrows). In some of the multinucleated giant cells, a characteristic but rare feature of the lung pathology in SARS, the immunohistochemical stainings were strongly positive (Figure 2b, arrowhead). Carbon pigments were not seen. These giant cells might represent those originated from syncytial formation of infected pneumocytes. Diffuse, weak, granular immunohistochemical signals were seen in carbon pigmentladen macrophages. These signals might represent background or scavengered proteins in these macrophages.

No specific histological changes had been identified in the SARS-CoV-infected intestine. While the virus can be isolated in some of our autopsy cases, 
no mucosal damage was observed. The lack of tissue damage is particularly striking in the terminal ileum biopsy from an active SARS patient with diarrhea. ${ }^{5}$ For the small intestine sections, the surface enterocytes showed positive cytoplasmic signals in both autopsy (Figure 3a) and biopsy (Figure 3b) specimens. Crypt cells were rarely positive. Macrophages in this organ were negative. The pattern of immunohistochemical-positive cells was consistent with the distribution of viral genome in other reports. ${ }^{16,17}$ The colon biopsy, however, was immunohistochemically negative for all the SARS-CoV proteins tested. Lymphoid follicles within these biopsies were also negative. In liver biopsies, no immunohistochemical signals were detected with the whole battery of antipeptide antibodies in any cell type. In situ hybridization studies using the probe to M-gene in the colon and liver sections were also negative (results not shown).

In those tissue sections showing positive signals for immunohistochemical staining, we further performed immunohistochemical studies using all other antibodies tested positive in SARSCoV-infected Vero E6 cells. These antibodies, including SARS-AbS18a, 7b, 24a, 20b, 19a, 15b, 9b and 11, all produced similar patterns of immunohistochemical staining in the lung and terminal ileum sections. The number of viral infected cells detected by each antibody was similar, judging semiqualitatively by the pattern of tissue distribution of the positive signals. The intensities of the immunohistochemical signals in each infected cells was correlated with those observed in Vero E6 cell culture (Table 1). These results suggested that, these peptides SARS 3a, 3b, 6, 7a and 9b corresponding to envelope, membrane, spike, nucleocapsid, were expressed in SARS-CoV infected cells in diseased organs.

\section{Coexistence of SARS-CoV Protein and RNA in the Cytoplasm of Infected Cells}

The cellular distribution of SARS-CoV protein and viral genome in immunohistochemical-positive lung and small intestine sections was further evaluated by immunofluorescence-fluorescence in situ hybridization analysis (Figure 4). SARS-CoV protein and viral genome were denoted by green (Figure 4-ii) and red fluorescence signals (Figure 4-iii), respectively. In the lung (Figure 4a) and small intestine (Figure 4b) sections, colocalization of immunohistochemical signals for SARS 3a and fluorescence in situ hybridization signals of SARS-CoV genome to the same cell was confirmed (Figure 4, arrows). Each of these signals was identified in the cytoplasm only. Only the cells infected with viral genome expressed the corresponding peptides, and vice versa.

\section{Discussion}

In this study, the tissue and cellular distributions of SARS-CoV hypothetical and structural proteins were evaluated in the autopsy and biopsy samples of SARS. We have also demonstrated the use of genome information that is generated from high throughput technologies for the translational research in diagnostic application. A new set of antipeptide antibodies was generated from the predicted ORFs of the structural and hypothetical proteins of the SARS-CoV. Immunohistochemical applications of these antibodies were validated on Vero E6 infected with SARS-CoV. Vero E6 is the prototype mammalian cell line that is used for SARS-CoV isolation in routine clinical microbiology investigations. ${ }^{3}$ In Vero E6 cells, positive cytoplasmic immunohistochemical signals were detected by
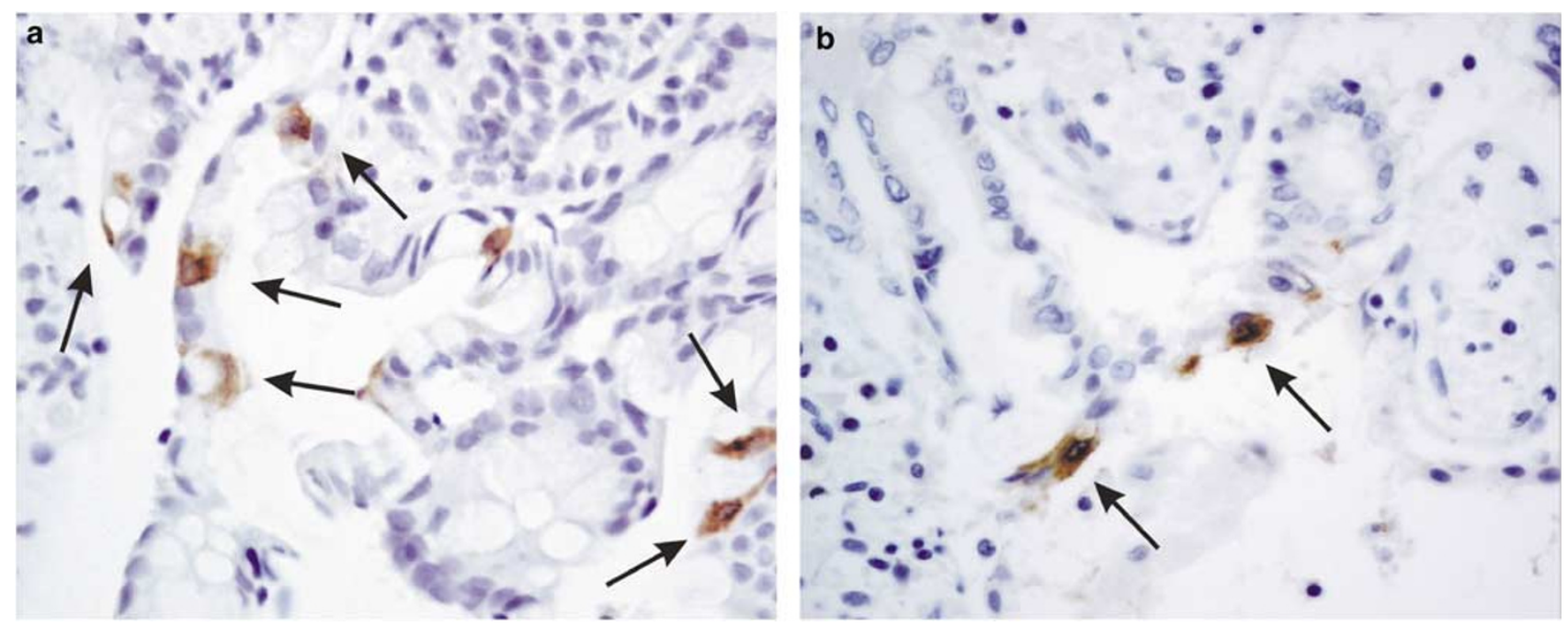

Figure 3 Immunohistochemical studies of antipeptide antibody SARS-AbS13a against the nucleocapsid protein on small intestine sections. SARS-CoV-infected cells were demonstrated by the strong cytoplasmic signals in surface enterocytes in both biopsy (a, arrows) and autopsy (b, arrows) specimens. The sections were counter-stained with hematoxylin $(\times 1000)$. 
i
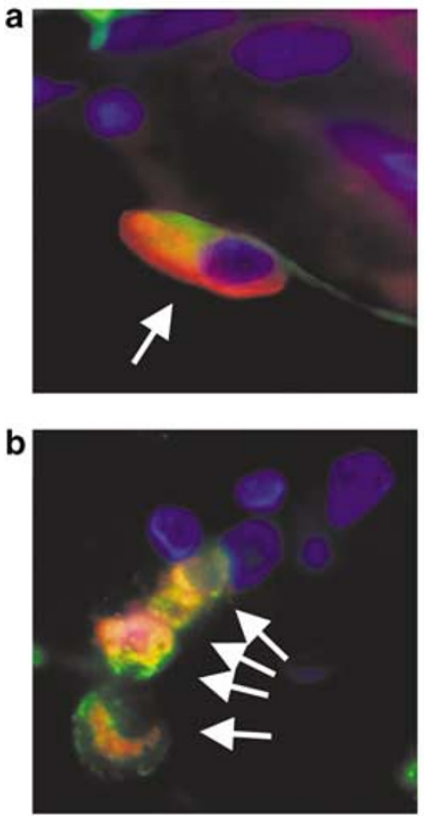

ii
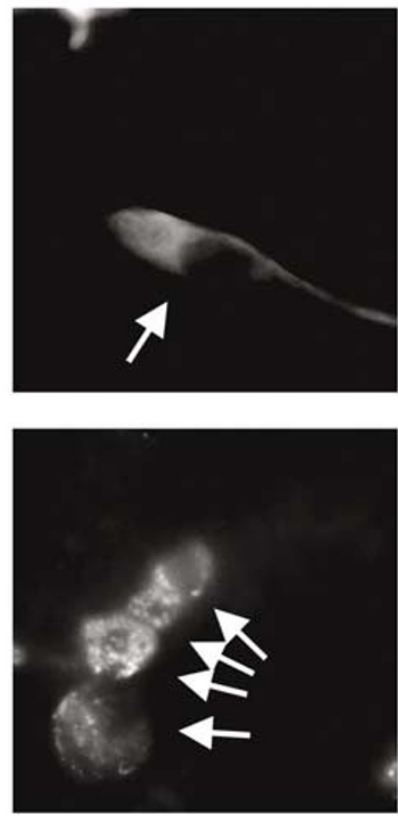

iii
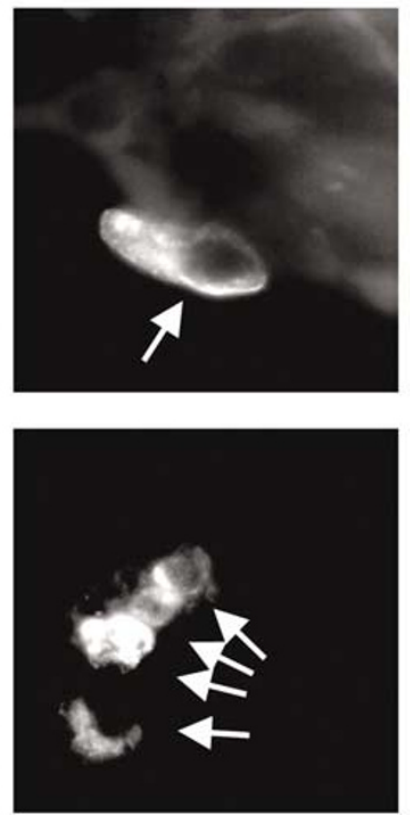

iv
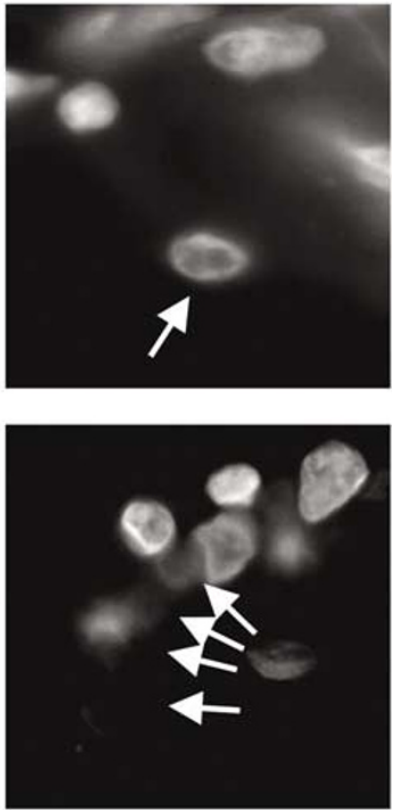

Figure 4 Immunofluorescence-fluorescence in situ hybridization of the hypothetical protein SARS 3a and SARS-CoV genome. SARS 3a (ii, green fluorescence) and viral genome (iii, red fluorescence) were detected using the antibody SARS AbS21a and a DNA-probe directed towards the $\mathrm{M}$ gene, respectively. The nuclei were counter-stained with 4,6-diamidino-2-phenylindole (iv). Pneumocytes (combined, a-i, arrows) and enterocytes (b-i, arrows) infected with the viral genome (iii, arrows) were positive for SARS 3a protein (ii, arrows). The background fluorescence of negative controls was limited to red blood cells and some connective tissue components.

at least one antibody from each predicted ORFs. The sequences of these peptides were unique and specific for the corresponding SARS-CoV protein. The specificities of these antipeptide antibodies were further determined experimentally with the suppression of immunohistochemical signals with excess corresponding peptides. In addition, in the study of tissue sections, only those cells with viral genome detected by fluorescence in situ hybridization were positive for immunohistochemical stainings for the antipeptide antibodies. These results demonstrated the existence of SARS-CoV known structural proteins and provide further evidence for the expression of those hypothetical proteins. The real identities of each of these hypothetical proteins require further investigations. However, our results indicated that at least the genome locations corresponding to the peptides selected for the putative ORFs were expressed. Further analyses of these hypothetical proteins by Western blotting and immunoprecipitation experiments in tissue culture models will be required to confirm the expression and molecular weights of these proteins, to look for possible post-translation modifications and to identify the host interacting partners. The feasibilities of these antibodies to be used in formalin-fixed paraffin-embedded sections of archival clinical specimens were established in the current study. Antibodies SARS-AbS13a, SARS-Abs-16b, SARSAbs-5a and SARS-AbS21a, against the nucleocapsid protein $(N)$, the membrane protein $(M)$, the spike protein (S) and a putative SARS 3a protein, respectively, appeared to provide strong immunohistochemical signals. These antibodies are potentially useful in the histological confirmation of SARS-CoV infection.

The existence of SARS-CoV proteins was further confirmed in clinical samples. Immunohistochemical staining was performed on all available tissues from nine autopsy cases of clinically confirmed SARS, two intestine biopsies of SARS patients and two liver biopsies. Positive cytoplasmic signals were observed in pulmonary pneumocytes and small intestinal surface enterocytes only. The existence of SARS-CoV in immunohistochemical-positive cells was proven by colocalization of the expressed proteins and viral genome in the same cells by dual immunofluorescence-fluorescence in situ hybridization. Both the lung pneumocytes and small intestine enterocytes expressed the full sets of viral proteins. Although lung and small intestine were the primary targets of SARS-CoV infection, the responses of these two tissues were quite different. Tissue damage and organization, in the pattern of diffuse alveolar damage, were commonly observed in the lungs of SARS. ${ }^{13,18-22}$ Syncytial cell formation is rarely observed although characteristic. Little tissue reaction is, however, observed in the small intestine autopsy and biopsy sections. ${ }^{5,13}$ Detailed studies of the expression levels and the interactions of viral proteins with host proteome may provide the answer. Much interest has been diverted to the 
study of known structural proteins, especially spike and nucleocapsid, in realizing the potentials of developing vaccines. The uniqueness of the hypothetical proteins to SARS-CoV, as compared phylogenetically to other known coronavirus, might be crucial to the clinical manifestation of SARS. At present, the functions of SARS-CoV hypothetical proteins and their roles in SARS pathogenesis are poorly understood. Recently, one of the putative proteins, SARS 7a was shown to interact with SARS 3a and the structural proteins in Vero E6. ${ }^{11,12}$ These findings highlighted the potential, undervalued functions of hypothetical proteins in the pathogenesis of SARS.

The negative immunohistochemical result in liver biopsies is interesting. Reverse transcriptase-polymerase chain reaction for the viral genome was positive while electron microscopy failed to demonstrate viral particles in the liver. ${ }^{14,22}$ In situ hybridization aiming at detecting the viral genome were all negative in these same specimens (result not shown). This might be due to the high sensitivity of polymerase chain reaction-based detection method that picked up the small number of viral genome in the scanty infected cells. Alternatively, serum contamination in the biopsy might explain the positive reverse transcriptase-polymerase chain reaction. It is, however, important to note that liver dysfunction was observed in SARS patients. ${ }^{14,23-25}$ A high proliferation index was clearly demonstrated in these liver biopsy specimens. ${ }^{14}$ Hence, liver metabolism must have been perturbed in these patients. The role of cytokines or other yet unknown paracrine mediators in causing liver dysfunction requires further clarification. A similar scenario is likely happening in the kidneys of SARS patients in which 'sensitive' reverse transcriptase-polymerase chain reaction is positive ${ }^{22}$ with functional disturbances of the renal function, yet viral particles, proteins and genome were not identified. ${ }^{26}$

We are beginning to understand how SARS-CoV enters its host cells. In various in vitro systems using the spike protein, the angiotensin-converting enzyme 2 (ACE2) 27-29 $^{27}$ and the C-type lectins, CD209 ${ }^{30}$ or CD209L, ${ }^{31}$ are identified as receptors of SARSCoV. The distribution of ACE2, by itself, cannot explain the tissue tropism of the virus. ${ }^{26}$ The ACE2 protein has a much wilder cellular distribution ${ }^{32}$ than even the most extensive, although putative and controversial, list of organs and cell types harboring the virus. ${ }^{17}$ In the current study, the dendritic cells were not positive for IHC signals of any of the viral proteins in various different tissues. Lymphoid follicles immediately adjacent to infected cells were negative. SARS-CoV may exist only in low titer in the dendritic cells. Parallel to the situations in human immunodeficiency virus-1 (HIV-1) and Mycobacterium tuberculosis ${ }^{33}$ the idea of dendritic cells as reservoir and immune mediator is attractive, the actual role of this cell type in SARS patients is not clear.
We have provided evidence for the presence of known structural proteins, including membrane, envelope, nucleocapsid and spike, as well as hypothetical proteins, SARS 3a, 3b, 6, 7a and 9b, in tissue culture model and in clinical specimens. With this full array of antibodies, only limited cell types were shown to harbor the SARS-CoV. The roles of these proteins and their interaction with the host genome and proteome are currently unknown. This set of antibodies will be useful in tissue diagnosis of SARS and in future studies of the in vitro models of SARS.

\section{Acknowledgements}

We thank Chit Chow, Cecilia Ho, and KW Suen for critical comments on the manuscript. The SARS project at Century Biotech Limited, is supported by Small Entrepreneur Research Assistance Program of the Innovation and Technology Commission, the Government of Hong Kong Special Administrative Region (Project no.: S/P675/03). Laboratory of AWIL was partially supported by Research Grant Council of Hong Kong Special Administrative Region (Project no.: CUHK4411/03 M).

\section{References}

1 WHO. Severe Acute Respiratory Syndrome (SARS) last accessed 2004, August 10.http://www.who.int/csr/ sars/en/ 2004.

2 Lee N, Hui D, Wu A, et al. A major outbreak of severe acute respiratory syndrome in Hong Kong. N Engl J Med 2003;348:1986-1994.

3 Tsang KW, Ho PL, Ooi GC, et al. A cluster of cases of severe acute respiratory syndrome in Hong Kong. N Engl J Med 2003;348:1977-1985.

4 Zhan J, Chen W, Li C, et al. Digestive system manifestations in patients with severe acute respiratory syndrome. Chin Med J (England) 2003;116:12651266.

5 Leung WK, To KF, Chan PK, et al. Enteric involvement of severe acute respiratory syndrome-associated coronavirus infection. Gastroenterology 2003;125:10111017.

6 Kuiken T, Fouchier RA, Schutten M, et al. Newly discovered coronavirus as the primary cause of severe acute respiratory syndrome. Lancet 2003;362:263-270.

7 Drosten C, Gunther S, Preiser W, et al. Identification of a novel coronavirus in patients with severe acute respiratory syndrome. N Engl J Med 2003;348:19671976.

8 Fouchier RA, Kuiken T, Schutten M, et al. Aetiology: Koch's postulates fulfilled for SARS virus. Nature 2003;423:240

9 Marra MA, Jones SJ, Astell CR, et al. The genome sequence of the SARS-associated coronavirus. Science 2003;300:1399-1404.

10 Rota PA, Oberste MS, Monroe SS, et al. Characterization of a novel coronavirus associated with severe acute respiratory syndrome. Science 2003;300:13941399. 
11 Tan YJ, Teng E, Shen S, et al. A novel severe acute respiratory syndrome coronavirus protein, U274, is transported to the cell surface and undergoes endocytosis. J Virol 2004;78:6723-6734.

12 Fielding BC, Tan YJ, Shuo S, et al. Characterization of a unique group-specific protein (U122) of the severe acute respiratory syndrome coronavirus. J Virol 2004;78:7311-7318.

13 Tse GM, To KF, Chan PK, et al. Pulmonary pathological features in coronavirus associated severe acute respiratory syndrome (SARS). J Clin Pathol 2004;57: 260-265.

14 Chau TN, Lee KC, Yao H, et al. SARS-associated viral hepatitis caused by a novel coronavirus: report of three cases. Hepatology 2004;39:302-310.

15 Chan PK, To KF, Lo AW, et al. Persistent infection of SARS coronavirus in colonic cells in vitro. J Med Virol 2004;74:1-7.

16 To KF, Tong JH, Chan PK, et al. Tissue and cellular tropism of the coronavirus associated with severe acute respiratory syndrome: an in situ hybridization study of fatal cases. J Pathol 2004;202:157-163.

17 Ding Y, He L, Zhang Q, et al. Organ distribution of severe acute respiratory syndrome (SARS) associated coronavirus (SARS-CoV) in SARS patients: implications for pathogenesis and virus transmission pathways. J Pathol 2004;203:622-630.

18 Cheung OY, Chan JW, Ng CK, et al. The spectrum of pathological changes in severe acute respiratory syndrome (SARS). Histopathology 2004;45:119-124.

19 Nicholls JM, Poon LL, Lee KC, et al. Lung pathology of fatal severe acute respiratory syndrome. Lancet 2003;361(9371):1773-1778.

20 Ding Y, Wang H, Shen H, et al. The clinical pathology of severe acute respiratory syndrome (SARS): a report from China. J Pathol 2003;200:282-289.

21 Franks TJ, Chong PY, Chui P, et al. Lung pathology of severe acute respiratory syndrome (SARS): a study of 8 autopsy cases from Singapore. Hum Pathol 2003;34: 743-748.

22 Hwang DM, Chamberlain DW, Poutanen SM, et al. Pulmonary pathology of severe acute respiratory syndrome in Toronto. Mod Pathol 2005;18:1-10.
23 Cui HJ, Tong XL, Li P, et al. Serum hepatic enzyme manifestations in patients with severe acute respiratory syndrome: retrospective analysis. World J Gastroenterol 2004;10:1652-1655

24 Chan HL, Leung WK, To KF, et al. Retrospective analysis of liver function derangement in severe acute respiratory syndrome. Am J Med 2004;116:566-567.

25 Wong WM, Ho JC, Ooi GC, et al. Temporal patterns of hepatic dysfunction and disease severity in patients with SARS. JAMA 2003;290:2663-2665.

26 To KF, Lo AW. Exploring the pathogenesis of severe acute respiratory syndrome (SARS): the tissue distribution of the coronavirus (SARS-CoV) and its putative receptor, angiotensin-converting enzyme 2 (ACE2). J Pathol 2004;203:740-743.

27 Wang P, Chen J, Zheng A, et al. Expression cloning of functional receptor used by SARS coronavirus. Biochem Biophys Res Commun 2004;315:439-444.

$28 \mathrm{Li} \mathrm{W}$, Moore MJ, Vasilieva N, et al. Angiotensinconverting enzyme 2 is a functional receptor for the SARS coronavirus. Nature 2003;426:450-454.

29 Hofmann H, Geier M, Marzi A, et al. Susceptibility to SARS coronavirus $S$ protein-driven infection correlates with expression of angiotensin converting enzyme 2 and infection can be blocked by soluble receptor. Biochem Biophys Res Commun 2004;319: 1216-1221.

30 Yang ZY, Huang Y, Ganesh L, et al. pH-dependent entry of severe acute respiratory syndrome coronavirus is mediated by the spike glycoprotein and enhanced by dendritic cell transfer through DC-SIGN. J Virol 2004;78:5642-5650.

31 Jeffers SA, Tusell SM, Gillim-Ross L, et al. CD209L (L-SIGN) is a receptor for severe acute respiratory syndrome coronavirus. Proc Natl Acad Sci USA 2004; 101(44):15748-15753.

32 Hamming I, Timens W, Bulthuis ML, et al. Tissue distribution of ACE2 protein, the functional receptor for SARS coronavirus. A first step in understanding SARS pathogenesis. J Pathol 2004;203:631-637.

33 van Kooyk Y, Geijtenbeek TB. DC-SIGN: escape mechanism for pathogens. Nat Rev Immunol 2003;3: 697-709. 